\title{
¿Qué significa en el ordenamiento español el derecho a «vivir con dignidad el proceso de la muerte»?
}

\author{
What does «the right to live the process of dying with \\ dignity» mean in Spanish law?
}

\author{
FERNANDO REY MARTÍNEZ*
}

Resumen: Se analizan en este texto diversos desarrollos normativos recientes en el ordenamiento jurídico español respecto de los derechos de la persona en el tramo final de su vida. En particular, se intenta aclarar el enigmático sentido de la emergente expresión «derecho a vivir con dignidad el proceso de la muerte» y, más allá de su actual configuración como derecho «legal» más que «constitucional», su posible catalogación como nuevo derecho fundamental.

Palabras clave: derecho a la protección jurídica de la vida - derecho a morir con dignidad - derecho a morir - derecho a vivir con dignidad el proceso de la muerte

Summary: Different recent regulatory developments in Spanish legal system are analyzed in this article concerning person's rights at the end of its life. In particular, the author attempts to explain the enigmatic sense of the new expression "the right to live the process of dying with dignity" and beyond its current configuration as a "legal" right-more than a constitutional oneAlso attempts to catalogue it as a new fundamental right.

Keywords: right to life's legal protection - right to die with dignity - right to die - right to live the process of dying with dignity 


\section{EL NUEVO Y ENIGMÁTICO DERECHO «A VIVIR}

CON DIGNIDAD EL PROCESO DE LA MUERTE»

En los últimos años, diversos Estatutos de Autonomía ${ }^{1}$, con sus correspondientes leyes de desarrollo ${ }^{2}$, así como un proyecto de ley estatal sobre los derechos de la persona ante el proceso del final de la vida, aprobado por el Consejo de Ministros de 17 de junio de 2011, han vuelto a poner sobre la mesa la cuestión del significado, en el ordenamiento jurídico español, de los derechos personales en relación con las decisiones sobre la salud e incluso la vida en el tramo final de la existencia humana. Esta normativa tiene un indudable interés, dentro y fuera de nuestras fronteras, tanto desde el punto de vista sustantivo (respecto de la dogmática de los derechos fundamentales), cuanto desde el punto de vista del reparto de competencias entre el Estado y los entes políticos sub-estatales (en el caso español, las Comunidades Autónomas).

El artículo 20 del Estatuto catalán, la primera disposición que se ha ocupado de estos asuntos con este nuevo enfoque, lleva la rúbrica o nomen iuris del «derecho a vivir con dignidad el proceso de la muerte», un derecho nuevo, de contenido un tanto enigmático. Inicialmente, el texto que aprobó el Parlamento catalán, antes de su aprobación definitiva por el Parlamento estatal, hablaba del derecho «a morir con dignidad». En el trámite parlamentario se modificó, creo que con buen criterio, el nombre.

La expresión «derecho a morir (con dignidad)» es tan expresiva como deliberadamente equívoca. Desde luego, no tiene un sentido preciso porque la muerte, más que un «derecho», es un «hecho», fatalmente inevitable por lo demás. En el influyente derecho norteamericano, el right to die se ha convertido en una rama del ordenamiento, de reciente creación, pero de desarrollo fulgurante, cuyo contenido, bastante variado (pues comprende disposiciones constitucionales, penales y de derecho de daños), remite a la regulación sobre el modo en que pueden adoptarse las decisiones en orden a rehusar el tratamiento médico

1 Aunque diversos Estatutos albergan derechos de decisión del paciente en el final de su existencia, sobre todo, como veremos, el derecho de instrucciones previas o testamento vital y el derecho a los cuidados paliativos, la expresión «derecho a vivir con dignidad el proceso de la muerte» se recoge expresamente por primera vez en el Estatuto catalán (ley orgánica 6/2006, de 19 de julio, de reforma del Estatuto de Autonomía de Cataluña). Por su parte, el Estatuto de Andalucía (ley orgánica 2/2007, de 19 de marzo, de reforma del Estatuto de Autonomía de Andalucía) se refiere, también en su artículo 20 , al derecho a la «dignidad ante el proceso de la muerte».

2 La primera norma, que marca la impronta es la ley $2 / 2010$, de 8 de abril, de derechos y garantías de la dignidad de la persona en el proceso de la muerte del Parlamento de Andalucía; a esta norma le sigue la Ley de Aragón 10/2011, de 24 de marzo, de derechos y garantías de la dignidad de la persona en el proceso de morir y de la muerte. $Y$ aunque todas las Comunidades Autónomas tienen leyes de desarrollo de la ley básica estatal 41/2002, de 14 de noviembre, reguladora de la autonomía del paciente y de derechos y obligaciones en materia de información y documentación clínica, puesto que son competentes en materia de protección de la salud, es interesante destacar también la Ley Foral de Navarra 17/2010, de derechos y deberes de las personas en materia de salud en la Comunidad Foral de Navarra porque en diversas partes de su articulado contempla derechos afines respecto de aquellos que estamos examinando en este estudio. 
de soporte vital o a la toma de decisiones sobre el final de la vida, pero la mayoría de autores se niega a comprender en él la eutanasia activa directa ni el suicidio asistido por un médico.

Con el «derecho a morir» se alude, pues, allí a lo que conceptualmente podemos denominar eutanasia activa indirecta (o medidas de doble efecto, paliativas y de acortamiento de la vida) y, sobre todo, eutanasia pasiva (o limitación de esfuerzo terapéutico). A veces, se utiliza como expresiones sinónimas «muerte natural» y/o «muerte con dignidad», frente a una deshumanizada «medicina de aparatos» ante la que las personas pueden convertirse finalmente en víctimas más que en beneficiarios. Su acta de nacimiento se remonta al caso In re Quinlan, decidido por la Corte Suprema de Nueva Jersey en 1976. Desde ese año, los tribunales estadounidenses han venido resolviendo decenas de asuntos y los legisladores de la mayor parte de los Estados han dictado alguna regulación al respecto. En la actualidad, como ha observado Alan Meisel, citando la Sentencia Thor v. Superior Court (1993) de la Corte Suprema de California ${ }^{3}$, hay «un creciente consenso judicial [...] que reconoce uniformemente el derecho del paciente a controlar su integridad corporal como el factor esencial en la determinación del equilibrio de intereses».

Entre nosotros, inicialmente el Parlamento catalán aprobó el proyecto de Estatuto con la denominación «derecho a morir con dignidad», pero, como se ha indicado, el Parlamento estatal la cambió por la de: «derecho a vivir con dignidad el proceso de la muerte», que es una expresión más afortunada por tres razones: i) porque describe mejor el contenido del derecho (que no es tanto un derecho a morir, cuanto a vivir dignamente el proceso final de la existencia); ii) porque no existe (por lo menos hasta el momento) un derecho constitucional a morir (o, más precisamente, a elegir morir), según ha subrayado en diversas ocasiones tanto el Tribunal Europeo de Derechos Humanos, en el asunto Pretty contra Reino Unido, de 29 de abril de 2002, como el Tribunal Constitucional español ${ }^{4}$; iii) y, por último, porque, de existir un derecho de este tipo,

3 MeISEL, Alan. The Right to Die. Segunda edición. Nueva York: Wiley Law Publications, 1995, p. 39. Incluye un cumulative supplement de 2003 en colaboración con Kathy CERMINARA. Los puntos principales de tal consenso serían los siguientes: i) Los pacientes capaces tienen un derecho constitucional a rehusar el tratamiento. ii) Los pacientes incapaces tienen los mismos derechos que los capaces. Los que deciden por subrogación de los pacientes incapaces deben intentar en primer lugar realizar las preferencias de estos expresadas antes de perder la capacidad de decidir; si tales deseos no fueran conocidos, las decisiones deberán adoptarse en función de la defensa de sus mejores intereses. iii) El proceso de toma de decisión debería ocurrir normalmente en sede médica, sin recurso a los tribunales (que solo deberían pronunciarse cuando se planteara algún problema). iv) La eutanasia activa y el suicidio asistido son conductas ética y jurídicamente distintas del rechazo al tratamiento médico que sostiene la vida.

4 En la STC 120/1990 se lee: «tiene [...] el derecho a la vida un contenido de protección positiva que impide configurarlo como un derecho de libertad que incluya el derecho a la propia muerte", tesis que se reitera en la STC 154/2002. Para un examen extenso de ambas decisiones, permítaseme la remisión al libro REY MARTíNEZ, Fernando. Eutanasia y derechos fundamentales. Madrid: CEPC/TC, 2008.

¿QUÉ SIGNIFICA

EN EL

ORDENAMIENTO

ESPAÑOL EL

DERECHO A

«VIVIR CON

DIGNIDAD EL

PROCESO DE LA

MUERTE»?

WHAT DOES «THE

RIGHT TO LIVE

THE PROCESS

OF DYING WITH

DIGNITY» MEAN

IN SPANISH LAW? 
dada su ligazón con el derecho a la protección de la vida, uno de los derechos fundamentales, su desarrollo correspondería al Estado y no a las Comunidades Autónomas.

Pero, además, en mi opinión, el concepto de «morir con dignidad» y el de «vivir con dignidad el proceso de la muerte» no coinciden. La expresión «morir con dignidad» es anfibológica. Con ella se suele aludir indistintamente hasta tres situaciones bastantes diferentes entre sí. En primer lugar, y quizás con carácter más habitual, se identifica con el control de los síntomas de la enfermedad terminal o aquella que produzca graves sufrimientos de tipo crónico. En este contexto se sitúan tanto las medidas y tratamientos de doble efecto (eutanasia activa indirecta — aquí se ubica el derecho a los cuidados paliativos-), como las limitaciones de esfuerzo terapéutico (eutanasia pasiva); ambas conductas son atípicas desde el punto de vista del derecho penal. En este punto se plantean los problemas más importantes y comunes en relación con el final de la vida humana. Cuando una persona normalmente formula su deseo de «morir con dignidad» está pidiendo, ante todo, una agonía sin dolor físico y en un entorno personal y médico adecuado. La importancia social de este sentido de la expresión «morir con dignidad» es inversamente proporcional a los problemas jurídicos que presenta, que son mínimos. El consenso en la comunidad jurídica sobre la validez jurídica de las medidas dirigidas al control del dolor, incluso aunque ello conlleve la aceleración de la muerte del paciente, o la interrupción de un tratamiento de soporte vital, es máximo.

En segundo lugar, con la expresión «morir con dignidad» se invoca la importancia de la voluntad del enfermo a la hora de adoptar las decisiones en el final de su vida. De ahí que el asunto de los documentos de instrucciones previas o voluntades anticipadas o testamento vital también se haya incorporado al contenido de este nuevo derecho de los Estatutos dentro de aquella rúbrica. Morir con dignidad significa que el protagonista del proceso que conduce a la muerte es el propio enfermo y no su entorno. Es la decisión del paciente y no la (normalmente) benevolente conspiración del médico y allegados del enfermo la que debe primar. En el contexto del nuevo paradigma de relación médico/ paciente de la autonomía del enfermo, basada en el principio del consentimiento informado, esta línea de argumentación también parece clara y tampoco parece suscitar demasiados problemas, o insalvables, en la práctica.

Pero por «derecho a morir con dignidad» en ocasiones también se quiere significar el suicidio asistido por médico y la eutanasia activa directa y voluntaria, como manifestaciones más radicales del control de los síntomas de la enfermedad terminal y de autonomía de la voluntad del paciente. Este sentido sí es problemático desde el punto de vista jurídico 
puesto que la facultad de poner a disposición la propia vida por uno mismo, ayudado por otros (suicidio asistido), o por terceros, aunque sea por móviles humanitarios y a petición del enfermo (eutanasia activa directa) siguen siendo castigadas por el artículo 143 del Código Penal.

Ahora bien, así como el derecho a morir con dignidad podría comprender, al menos teóricamente, los tres sentidos descritos, el «derecho a vivir con dignidad el proceso de la muerte» solo abarcaría los dos primeros sentidos mencionados, pero de ninguna manera el tercero. Esta es la hipótesis exploratoria de este trabajo, la determinación del contenido de este nuevo derecho, con un sentido de menor contenido que el derecho a morir con dignidad, pero más coherente con la prohibición penal en nuestro ordenamiento de la eutanasia y el suicidio asistido. Sin duda, es preciso un intento de clarificación conceptual. En España, este nuevo derecho lo introducen los Estatutos de Autonomía catalán y andaluz, esto es, se trata de un derecho de clara naturaleza legislativa. Por el momento, es un derecho legal y no constitucional. De ahí que analicemos, a continuación, las normas autonómicas que configuran su contenido. Sin embargo, una cuestión dogmática interesante que abordaremos tras dicho análisis es si estamos en presencia de un nuevo derecho que pudiera llegar a tener, incluso, estatura constitucional, es decir, que podría reconocerse expresamente en una eventual reforma constitucional futura (en España o en cualquier otro lugar), o que podría, incluso, mientras tanto, deducirse de la tabla de derechos ya existente. En otras palabras, nos plantearemos si se trata de un derecho legal llamado a convertirse en derecho constitucional fundamental.

\section{LA CUESTIÓN EN LA ÚLTIMA OLEADA \\ DE REFORMAS ESTATUTARIAS}

Las modificaciones recientes de algunos Estatutos de Autonomía, empezando por el de Cataluña, han reconocido, dentro de la tabla de derechos que incorporan, el derecho a «vivir con dignidad el proceso de la propia muerte». El texto de Estatuto que aprobó el Parlamento de Cataluña el 30 de septiembre de 2005 incluyó, un artículo, el vigésimo, bajo la rúbrica «derecho a morir con dignidad», con el siguiente contenido:

1. Todas las personas tienen derecho a vivir con dignidad el proceso de su muerte. 2. Todas las personas tienen derecho a expresar su voluntad de forma anticipada para dejar constancia de las instrucciones sobre las intervenciones y tratamientos médicos que puedan recibir, que deben ser respetadas, en los términos que establezcan las leyes, especialmente por el personal sanitario cuando no estén en condiciones de expresar personalmente su voluntad. 
Esta disposición tenía una redacción deliberadamente ambigua en su rúbrica, «derecho a morir con dignidad», y un contenido incierto, que parecía admitir el reconocimiento en ciertos casos de la eutanasia directa (y así fue advertido en el debate partidista y por los medios de comunicación). Al menos simbólicamente, ya que la prohibición penal seguía vigente, así como la ley estatal básica sobre derechos del paciente, que establecía - y establece - un marco bien preciso de límites, también para los documentos de instrucciones previas o voluntades anticipadas a los que se refiere el apartado segundo. La norma no podía, obviamente, despenalizar la eutanasia directa en ningún caso, pero su redacción era simpática a esta posibilidad y parecía animar (dada la situación de las mayorías políticas del momento en Cataluña y en Madrid) una promesa de reforma estatal en clave despenalizadora. En este sentido, fue leída como un triunfo político (de ningún modo jurídico) de los partidarios de la despenalización de la eutanasia 5 . Quizás por ello tal redacción no prosperó en la tramitación de la reforma del Estatuto en el Congreso de los Diputados. El debate giró, a instancias del nuevo acuerdo entre el grupo socialista y el convergente, hacia el reconocimiento del derecho a recibir adecuados tratamientos paliativos, lo cual supuso una inteligente manera de desactivar el problema planteado. La relación inversamente proporcional entre la calidad de los cuidados paliativos y la necesidad de eutanasia activa es un argumento que aparece de modo crónico en el debate sobre la eutanasia.

La redacción que finalmente se aprobó en el Estatuto catalán suprime la indefinida rúbrica «derecho a morir con dignidad» y la sustituye (también en el artículo 20) por la expresión «derecho a vivir con dignidad el proceso de la muerte» ${ }^{6}$, que también es ambigua, pero que se refiere no a un supuesto «derecho a morir con dignidad», sino, en positivo, por decirlo así, a «vivir con dignidad» el proceso de la propia muerte. Su ubicación sistemática en el mismo apartado donde se reconoce el derecho del paciente a los cuidados paliativos permite precisar o tematizar ya, en alguna medida, su campo de aplicación. A pesar de este intento de suavizar y reconducir la cuestión ${ }^{7}$, es dudoso

5 Hay que recordar que los dos socios minoritarios del tripartito catalán, los republicanos y los comunistas/verdes eran entusiastas partidarios de la despenalización de la eutanasia (sobre todo, los últimos) y que el socio mayoritario, el PSOE, había incluido en el programa de las últimas elecciones generales la promesa de la despenalización, aunque la aparcó para la segunda legislatura, en la que tampoco la promovió. En mi opinión, el sentido político de este inicial artículo 20 era el de presionar al Gobierno de Madrid para que promoviera una reforma del Código Penal en esta dirección, conscientes las autoridades políticas catalanas de su manifiesta carencia de competencia.

6 Que vuelve a aparecer en el apartado primero: «Todas las personas tienen derecho a recibir un adecuado tratamiento del dolor y cuidados paliativos integrales y a vivir con dignidad el proceso de su muerte». El párrafo segundo, sobre el documento de voluntades anticipadas, se mantiene intacto en la redacción finalmente aprobada del Estatuto (artículo 20.2).

7 Es significativa en este sentido la declaración de un diputado de CiU (Convergència Democràtica de Catalunya i Unió Democràtica de Catalunya), J. Sánchez Llibre, tras la aprobación de la enmienda transaccional que modificó la redacción original: «Queremos dar este mensaje de tranquilidad, de serenidad a todos los ciudadanos y ciudadanas de Cataluña. A través de estas modificaciones y de 
que una Comunidad Autónoma pudiera regular aspectos concernientes a derechos tan personales (vida, integridad, etcétera) como los aquí invocados.

No obstante, la impronta del Estatuto catalán se deja sentir en este punto, como en tantos otros, en el nuevo Estatuto andaluz, concretamente en su artículo $20^{8}$. Con una redacción más sencilla, el contenido del precepto es prácticamente idéntico. La rúbrica del precepto añade la expresión «testamento vital» a la de «dignidad ante el proceso de la muerte». Técnicamente no parece del todo correcto porque no distingue los conceptos de «testamento vital» (al que se refiere en el nomen iuris del precepto) y «voluntades anticipadas» (que es a lo que se refiere de verdad en el apartado primero) y porque la yuxtaposición, por ese orden además, de «testamentos vitales» $\mathrm{y}$ «dignidad ante el proceso de la muerte» es poco elegante. Los testamentos vitales pueden ser una parte de los documentos de voluntades anticipadas que, a su vez, pueden entenderse como un elemento necesario para que un enfermo inconsciente sea tratado en el momento de su muerte con dignidad ${ }^{9}$. De modo que habría una relación de especie (testamento vital) a género (dignidad en el proceso de la muerte) que hubiera aconsejado imitar aun más al Estatuto catalán para especificar en la rúbrica tan solo la expresión «dignidad en el proceso de la muerte». $\mathrm{O}$ una expresión similar: la redacción andaluza solo cambia respecto de la catalana al reclamar que el derecho a la dignidad en el proceso de la muerte haya de ser «plena» ${ }^{10}$, a mi juicio, un simple pleonasmo porque, evidentemente, la dignidad solo puede presentarse de modo pleno, como la misma vida: se tienen o no. Tertium non datur.

Así pues, al Estatuto andaluz le ocurre en este punto como a algunos investigadores noveles, que pueden ser buenos u originales, pero no ambas cosas a la vez. En cualquier caso, la innovadora línea emprendida por el estatuyente catalán en relación con la identificación de un «derecho a vivir con dignidad el proceso de la muerte» solo ha sido seguida por el andaluz y no por el resto de Estatutos reformados ${ }^{11}$, pero todos ellos, siguiendo su estela, recogen el contenido finalmente atribuido a esta expresión: el derecho a recibir cuidados paliativos, esto es, el derecho frente al dolor físico, y el derecho a otorgar

estas enmiendas podemos afirmar categóricamente que este Estatut ya no configura la eutanasia ni el suicidio asistido" (La Vanguardia, 11 de marzo de 2006, p. 19).

8 «1. Se reconoce el derecho a declarar la voluntad vital anticipada que deberá respetarse, en los términos que establezca la ley. 2. Todas las personas tienen derecho a recibir un adecuado tratamiento del dolor y cuidados paliativos integrales y a la plena dignidad en el proceso de su muerte".

9 Sobre este particular, puede consultarse: DE MONTALVO, Federico. Testamento vital y Constitución: los límites del testamento vital. Madrid: Cátedra de Bioética, Universidad Pontificia Comillas, 2009.

10 También el artículo 4 a) de la ley aragonesa 10/2011, se refiere, dentro de los «principios básicos», a «la plena dignidad de la persona en el proceso de morir», reclamando además para ello "pleno respeto".

11 Que han sido los de Valencia, Cataluña, Andalucía, Castilla y León, Baleares, Aragón y Extremadura.

¿QUÉ SIGNIFICA

EN EL

ORDENAMIENTO

ESPAÑOL EL

DERECHO A

«VIVIR CON

DIGNIDAD EL

PROCESO DE LA

MUERTE»?

WHAT DOES «THE

RIGHT TO LIVE

THE PROCESS

OF DYING WITH

DIGNITY» MEAN

IN SPANISH LAW? 
un escrito de instrucciones previas, voluntades anticipadas o testamento vital. Así, por ejemplo, el artículo 25.4 de la reforma del Estatuto de Islas Baleares recoge el «nuevo derecho estatutario» a los cuidados paliativos y a «declarar su voluntad vital anticipada», al igual que los textos catalán y andaluz, pero sin la referencia más abierta y problemática al derecho a vivir con dignidad el proceso de la muerte. El artículo 25.4 reza: «Todas las personas tienen derecho a un adecuado tratamiento del dolor y a cuidados paliativos, así como a declarar su voluntad vital anticipada que deberá respetarse en los términos que establezca la ley». Por su parte, el artículo 14.4 del nuevo Estatuto de Aragón no solo no recoge la problemática alusión al derecho a vivir con dignidad el proceso de la propia muerte, sino tampoco el derecho a los cuidados paliativos; se refiere únicamente al documento de voluntades anticipadas: «Todas las personas podrán expresar su voluntad, incluso de forma anticipada, sobre las intervenciones y tratamientos médicos que deseen recibir, en la forma y con los efectos previstos en las leyes».

He tenido oportunidad de comprobar en primera persona, por haber participado en el proceso de redacción técnica de la reforma del Estatuto de Castilla y León, que esta cuestión es, de suyo, políticamente conflictiva. En efecto, la redacción de lo que más tarde sería el artículo 13.2.f), relativo al derecho «a recibir tratamientos y cuidados paliativos adecuados» de la Propuesta de reforma del Estatuto aprobada por las Cortes de Castilla y León el 29 de noviembre de 2006, causó uno de los momentos más críticos y tensos del debate en el seno de la ponencia parlamentaria extraordinaria encargada de la reforma porque el Grupo parlamentario popular, mayoritario, no quería a ningún precio que este texto se pudiera entender como una puerta abierta al reconocimiento (aunque fuera político y un tanto engañoso y «publicitario») de la eutanasia.

De modo que, aunque el debate de los nuevos Estatutos no ha hecho avanzar el reconocimiento jurídico de la eutanasia (por la propia limitación de su factura institucional no podía hacerlo), sí ha provocado, sin embargo, en este punto un interesante resultado: ha consagrado como «nuevo» derecho (dentro de la reciente y discutida categoría de los «derechos estatutarios») uno de naturaleza prestacional, cuyo contenido esencial es triple: el derecho a los cuidados paliativos, el derecho frente al dolor físico ${ }^{12}$ y el derecho de consentimiento informado en el proceso del final de la vida, que incluye el derecho a rechazar el tratamiento, incluso de soporte vital, y el derecho a otorgar voluntades anticipadas o instrucciones previas. 
Pero así como ha sido el Estatuto de Autonomía catalán el primero que ha reconocido expresamente este nuevo derecho, la primera configuración legislativa autonómica de desarrollo ha sido la andaluza. En efecto, la ley 2/2010, de 8 de abril, de derechos y garantías de la dignidad de la persona en el proceso de la muerte del Parlamento de Andalucía, ha venido a desarrollar el artículo 20 de su respectivo Estatuto de Autonomía. En mi opinión, los aspectos principales de la Ley son los siguientes:

1. No se refiere de ningún modo a la eutanasia activa directa (como no podía ser de otra manera).

2. El rechazo del tratamiento, la limitación de medidas de soporte vital y la sedación paliativa (esto es, lo que podría denominarse eutanasia pasiva y activa indirecta) se reconocen expresamente. El legislador andaluz tiene muy en cuenta la experiencia del caso Inmaculada Echevarría ${ }^{13}$, que es determinante para entender el sentido político de la norma.

3. La ley consolida el derecho de los pacientes a recibir cuidados paliativos integrales de alta calidad (en el domicilio que designen dentro de la Comunidad andaluza: artículo 12) y a redactar un documento en el que hagan constar sus deseos y preferencias de tratamiento para el caso de que no pudieran en el futuro decidir por sí mismas, así como a designar a quien tome las decisiones en su lugar (artículo 9).

4. Se precisan en el Título III (artículos 17 a 21) los deberes de los profesionales sanitarios que atienden a los pacientes en el proceso de muerte y atribuye un conjunto de obligaciones para instituciones sanitarias, públicas o privadas (de fondo, el legislador no olvida que Inmaculada Echevarría tuvo que ser desplazada desde un hospital privado a otro público para proceder a su desconexión y sedación dada la negativa de los responsables del primero a llevar a cabo esas acciones).

5. Se introducen otros derechos prestacionales, que no me parecen precisamente de rango menor: el apoyo a las familias de la persona en situación terminal, incluyendo la atención al duelo (artículo 24), y la provisión de una habitación individual para este tipo de casos (en la que podrán estar acompañados permanentemente por un familiar o persona allegada: artículo 26).

6. Por último, se facilita el acceso de todos los centros e instituciones sanitarias a un Comité de Ética Asistencial con funciones

¿QUÉ SIGNIFICA

EN EL

ORDENAMIENTO

ESPAÑOL EL

DERECHO A

«VIVIR CON

DIGNIDAD EL

PROCESO DE LA

MUERTE»?

WHAT DOES «THE

RIGHT TO LIVE

THE PROCESS

OF DYING WITH

DIGNITY» MEAN

IN SPANISH LAW? 
de asesoramiento en los casos de decisiones clínicas que planteen conflictos éticos (artículo 27).

La ley andaluza ha inspirado el contenido de la Ley de Aragón 10/2011, de 24 de marzo, de derechos y garantías de la dignidad de la persona en el proceso de morir y de la muerte. Curiosamente, el Estatuto de Aragón, que es uno de los reformados últimamente, no contempla disposición alguna relativa a este tipo de derechos, lo cual no quiere decir que carezca de menor título competencial que otras Comunidades (el título genérico de protección de la salud es suficientemente habilitante). El título de la ley aragonesa vuelve a modificar parcialmente el nomen iuris hasta ese momento utilizado. Ya no se habla del «derecho a vivir con dignidad el proceso de la muerte», como en la norma catalana, ni del «derecho de dignidad ante el proceso de la muerte», como en la norma andaluza. iPara el intérprete resulta más fácil determinar el contenido de este nuevo derecho que su denominación! El título de la ley aragonesa coincide con la andaluza en mencionar el derecho «de dignidad» (la aragonesa habla también de «garantía») y no se refiere al derecho «a vivir con dignidad» el proceso de la muerte; además, alude al «proceso de la muerte», como las otras normas, pero añade (sin que sea fácil advertir, de entrada, la diferencia) el proceso «de morir». A pesar de estas variaciones terminológicas, que tendremos oportunidad de valorar más adelante, el contenido de la norma aragonesa coincide casi punto por punto con la norma andaluza. Entre las peculiaridades más notables, sin embargo, procede señalar las siguientes:

1. La ley aragonesa contiene una regulación algo diferente en relación con los menores de edad. No solo asume la tesis normativa general española de la equiparación del menor de 18 años, pero mayor de 16 con los mayores de edad, sino que va algo más allá puesto que el artículo 11.3 de la ley, de modo sorprendente, en mi opinión, permite a los niños mayores de 14 años (con capacidad de decidir) «prestar por sí mismos consentimiento informado y otorgar documento de voluntades anticipadas», con la asistencia de uno de sus padres o del tutor. E incluso el menor de 14 años, si tiene «suficiente juicio para entender el alcance de la intervención sanitaria propuesta», podrá otorgar el consentimiento informado, si bien en este caso necesitará «autorización conjunta de los titulares de la autoridad familiar o del tutor» (artículo 11.2). Este punto de la regulación (quizá inspirado en la relevancia que el derecho foral aragonés otorga tradicionalmente a la voluntad de los menores) me parece un auténtico despropósito.

2. La disposición adicional segunda ordena al Gobierno de Aragón evaluar la aplicación de la ley en el plazo de un año desde su entrada en vigor y analice «la forma en que la ciudadanía aragonesa enfrenta y vive el proceso de su muerte». 
3. Por último, la disposición adicional tercera ordena al Gobierno de Aragón elaborar un plan de cuidados paliativos en el plazo máximo de un año.

En definitiva, Estatutos y leyes autonómicas de desarrollo han ido conformando el sentido y alcance de este nuevo derecho de las personas en relación con el proceso de su muerte. En este contexto, se explica el intento del Gobierno central de dictar una ley con vocación de ser básica, es decir, de extender los nuevos derechos a todos los españoles y de servir como mínimo denominador común normativo de la materia en todo el territorio.

\section{EL PROYECTO DE LEY ESTATAL REGULADORA DE LOS DERECHOS DE LA PERSONA ANTE EL PROCESO DEL FINAL DE LA VIDA}

El Gobierno (socialista) de la Nación aprobó el 17 de junio de 2011 el proyecto de ley estatal reguladora de los derechos de la persona ante el proceso del final de la vida. Todo parece indicar, sin embargo, que este proyecto, o cualquier versión de él, no llegará finalmente a ser aprobado ya que el final de la legislatura provocó su caducidad y el partido que sostiene al nuevo gobierno, conservador, lo rechazó frontalmente. Esto no quiere decir que los derechos más importantes relativos al proceso del final de la vida no se reconozcan en el ordenamiento español, puesto que tales derechos han sido regulados por la ley 41/2002, de 14 de noviembre, de autonomía del paciente y de derechos y obligaciones en materia de información y documentación clínica (que, en gran medida, incorpora el Convenio del Consejo de Europa para la protección de los derechos humanos y la dignidad del ser humano respecto de las aplicaciones de la biología y la medicina, suscrito en Oviedo el 4 de abril de 1997 — ha entrado en vigor en España el 1 de enero de 2000—).

El proyecto era un texto corto, de tan solo 19 artículos. Lo principal de su regulación puede, quizá, contraerse a estas ideas:

1. El proyecto se refiere exclusivamente a las personas «en el proceso final de su vida», que comprende (artículo 2.2) las personas en situación terminal ${ }^{14}$ o en situación de agonía ${ }^{15}$, pero, eso sí, tanto del ámbito asistencial público como privado (artículo 2.1).

14 «Entendiendo por tal la presencia de una enfermedad, incurable y progresiva, en estado avanzado, sin posibilidades razonables de respuesta al tratamiento específico, con un pronóstico de vida limitado a semanas o meses y en la que pueden concurrir síntomas intensos que requieren una asistencia paliativa especializada".

15 «Definida como la fase gradual que precede a la muerte y que se manifiesta clínicamente por un deterioro físico grave, debilidad extrema, trastornos cognitivos y de consciencia, dificultad de relación y de ingesta y pronóstico vital de pocos días».

¿QUÉ SIGNIFICA EN EL

ORDENAMIENTO

ESPAÑOL EL

DERECHO A

«VIVIR CON

DIGNIDAD EL

PROCESO DE LA

MUERTE»?

WHAT DOES «THE

RIGHT TO LIVE

THE PROCESS

OF DYING WITH

DIGNITY» MEAN

IN SPANISH LAW? 
2. Los derechos que prevé para los pacientes mayores de 16 años ${ }^{16}$ son:

a) Derecho a la información asistencial (artículo 3), clara y comprensible, sobre su estado real de salud, expectativas de vida y calidad de la misma y medidas terapéuticas y paliativas aplicables. El enfermo tiene derecho a rechazar la oferta de información y, en ese caso, deberá designar una persona que acepte recibirla, haciendo constar esta situación en la historia clínica (artículo 3).

b) Derecho a la toma de decisiones (artículo 4): podrán rechazar las intervenciones propuestas por los profesionales sanitarios para su tratamiento, aunque ello pudiera acortar su vida o ponerla en peligro inminente (salvo peligro para la salud pública en los términos del artículo 9.2 de la ley 41/2002). El apartado segundo de este artículo cuarto introduce la cautela de que el rechazo al tratamiento médico «no supondrá menoscabo alguno en la atención sanitaria de otro tipo», sobre todo la paliativa. El consentimiento informado (cuyo contenido es, por supuesto, revocable) deberá ser expreso, constar por escrito e incorporarse a la historia clínica.

Por su parte, los profesionales sanitarios deberían adecuar el esfuerzo terapéutico (artículo 15.2) de modo proporcional a la situación del paciente, evitando la adopción o mantenimiento de intervenciones y medidas de soporte vital carentes de utilidad clínica, en atención a la cantidad y calidad de vida futuras del paciente; en este caso, la adecuación del esfuerzo terapéutico requerirá del juicio coincidente de, al menos, otro médico que participe en la atención sanitaria y se adoptará tras informar al paciente o a su representante, tomando en consideración su voluntad y oído el criterio profesional del personal de enfermería responsable de los cuidados (artículo 15.2).

El consentimiento por representación en casos de incapacidad (artículo 6) se prestará por este orden ${ }^{17}$ : i) persona designada como representante en el documento de instrucciones previas; ii) representante legal; iii) cónyuge o persona vinculada por análoga relación de afectividad; iv) parientes de grado más próximo y, dentro del mismo grado, pariente de mayor edad. El apartado quinto del artículo sexto plantea qué ocurre cuando

16 La norma no introducía especialidades respecto de las reglas generales sobre la mayoría de edad para adoptar decisiones de salud a partir de los 16 años de la ley 41/2002.

17 «Siempre buscando el mayor beneficio y respeto a la dignidad de los pacientes que se encuentren en situación de incapacidad, teniendo en cuenta en todo caso las manifestaciones y criterios que el paciente hubiera realizado con anterioridad a la situación de incapacidad” (artículo 6.4). 
exista contradicción entre lo manifestado por el paciente y por su representante: deberá prevalecer siempre la voluntad del paciente, debiendo actuar los profesionales sanitarios conforme a la misma.

c) El derecho a otorgar el documento de instrucciones previas (artículo 7), así como a revocarlo o modificarlo en cualquier momento (artículo 8). Toda persona mayor de edad y con plena capacidad de obrar tiene derecho a otorgar el documento de instrucciones previas (ya previsto en el artículo 11 de la ley 41/2002, que también prevé la creación de un registro específico), cuyo contenido puede ser: i) manifestar anticipadamente su voluntad sobre los cuidados y el tratamiento asistencial que desea recibir en el proceso del final de su vida; ii) designar un representante y determinar sus funciones, a las que este deberá atenerse, velando para que se cumpla la voluntad del representado para las situaciones clínicas que este previó y, en caso de que no las hubiera previsto, deberá tener en cuenta las opciones deducibles de las expresamente recogidas en el documento y los valores del otorgante.

d) Derecho al tratamiento del dolor (artículo 9) mediante una «atención idónea», que incluya: i) «cuidados paliativos integrales de calidad ${ }^{18}{ }_{»}$; ii) asistencia domiciliaria en los cuidados paliativos que se precisen; y iii) la sedación paliativa si el dolor es refractario (es decir, resistente a cualquier otro tratamiento).

e) Derecho al acompañamiento (artículo 10), incluyendo el auxilio espiritual de elección, pero siempre que resulte compatible con el conjunto de medidas sanitarias necesarias para ofrecer una atención de calidad. El artículo 19 alberga no como un derecho de los pacientes, sino una obligación de las administraciones sanitarias, el apoyo emocional durante el proceso del final de la vida a los pacientes y a sus familias.

f) Derecho a la intimidad personal y familiar (artículo 11), un derecho de contenido amplio, que la norma concreta en dos aspectos: i) el derecho a una habitación individual durante su estancia (aunque la norma matiza que «de acuerdo con los medios de que dispongan los centros e instituciones

18 «Entendidos como un conjunto coordinado de intervenciones sanitarias dirigidas, desde un enfoque integral, a la prevención y alivio del sufrimiento de las personas que se encuentran en el proceso final de su vida, por medio de la identificación temprana y la evaluación y tratamiento del dolor y otros problemas físicos y psíquicos» (artículo 9.2.a).

¿QUÉ SIGNIFICA

EN EL

ORDENAMIENTO

ESPAÑOL EL

DERECHO A

«VIVIR CON

DIGNIDAD EL

PROCESO DE LA

MUERTE»?

WHAT DOES «THE

RIGHTTO LIVE

THE PROCESS

OF DYING WITH

DIGNITY» MEAN

IN SPANISH LAW? 
sanitarias»); y ii) la protección de datos relacionados con su atención sanitaria, historia clínica e instrucciones previas.

El proyecto presentaba algunos aspectos discutibles o mejorables, como, por ejemplo, su rango (por la afectación de algunos derechos fundamentales, como el derecho a la integridad personal —artículo $15 \mathrm{CE}$, debiera ser orgánica en algunos aspectos y no simplemente ordinaria-); o que no se refiriera en absoluto a la objeción de conciencia de los profesionales sanitarios, entre otros temas. Pero, en definitiva, el proyecto nos resulta útil, leído conjuntamente con la normativa autonómica, para determinar qué se entiende en el ordenamiento español cuando se habla de los derechos en el proceso del final de la vida, que es el objeto principal de este análisis.

\section{CONCLUSIONES: TERMINOLOGÍA Y CONTENIDO DE LOS DERECHOS DE LA PERSONA EN EL PROCESO DE MORIR}

En primer lugar, las vacilaciones terminológicas que hemos encontrado en la normativa española requieren cierta clarificación. Las dudas sobre las palabras suelen manifestar dudas sobre los conceptos. El centro de gravedad de todas las denominaciones legales son las palabras «dignidad» y «muerte». Pues bien, ya hemos descartado la expresión «derecho a morir con dignidad» porque es equívoca e incluye en su significado algún sentido contrario al ordenamiento. En la medida en que este nuevo derecho otorga a sus titulares auténticas facultades judicialmente exigibles cuyo contenido, además, puede modular libremente, esto es, puesto que se trata de un genuino derecho subjetivo, es preferible la expresión «derecho» a la más objetiva noción de «garantía», que subraya la técnica de protección y no tanto la voluntad del titular.

¿Qué expresión es más precisa: «derecho a vivir con dignidad el proceso de la muerte» o «derecho de dignidad ante el proceso de la muerte»? La remisión a la idea de dignidad es evocadora, pero, al mismo tiempo, conceptualmente algo perturbadora en la medida en que los derechos fundamentales protegen bienes constitucionales como la libertad, la igualdad, la vida, la salud, la intimidad, el honor, la participación en el proceso político, etcétera, pero no directamente la dignidad como un bien específico. Libertad, igualdad, vida, etcétera, son manifestaciones o concreciones de la dignidad humana, fundamento del Estado y de la sociedad (artículo 10.1CE). Pero, por otrolado, es cierto que el contenido del nuevo derecho que estamos examinando es, en parte, un derecho de libertad en un contexto determinado (decisiones relacionadas con la propia muerte), que comprende facultades como otorgar o no un documento de voluntades anticipadas, con el contenido que se desee (con la limitación de la prohibición de eutanasia activa directa 
y de suicidio asistido), o de rechazar un tratamiento, incluso de soporte vital; pero, en parte, este nuevo derecho tiene un contenido prestacional: el más relevante, el derecho a los cuidados paliativos, el derecho frente al dolor físico derivado de una enfermedad. La idea de dignidad permitiría englobar ambos aspectos, el de libertad y el prestacional o social, pero no es menos cierto que también otros derechos tienen un contenido de libertad y prestacional (pensemos, por ejemplo, en el derecho de educación), sin que haya sido necesario recurrir a la idea de la dignidad en el proceso de la educación o similar. Ciertamente, las regulaciones autonómicas españolas han intentado regular lo que habitualmente se denomina «el ideal de una muerte digna», que es una expresión que plantea problemas porque, iqué ocurre con las personas que no mueren así, son personas que mueren indignamente?

El derecho en examen tiene un contenido especialmente heterogéneo también por lo que se refiere a sus titulares porque comprende pacientes terminales ${ }^{19}$, pero también personas sanas que puedan decidir escribir o no sus voluntades anticipadas. En realidad, es el proceso de la propia muerte (cercana o futura) lo que da unidad a todos los derechos que engloba.

Recapitulando. Para determinar con precisión la denominación del nuevo derecho, la idea de «derecho» debe mantenerse y también la del «proceso de la muerte» (o la equivalente del «proceso de morir» - pero no, sin más, de un eventual derecho, faústico, frente a la muerte-); la evocación de la dignidad, aunque sonora, puede considerarse redundante. Personalmente, propondría la denominación (por cierto muy cercana a la que preveía el proyecto de ley del Estado) de los derechos en el proceso de morir (el proyecto de ley estatal suprimía la mención a la muerte y hablaba, eufemísticamente, del «proceso final de la vida» — expresión que también podría aceptarse-; personalmente encuentro más potente, más expresiva, la alusión a la muerte). No obstante, soy consciente de que en el ordenamiento español ha entrado con fuerza la idea del derecho a «vivir con dignidad» o del «derecho de dignidad» en el proceso de la muerte, de modo que no es probable un cambio de denominación.

¿Qué contenido tendría ese nuevo derecho? Tanto la normativa autonómica, como el proyecto de ley estatal ofrecen pistas claras sobre su sentido y alcance. Desde luego, no incluye el derecho a la eutanasia activa directa y/o al suicidio asistido. Su contenido pasa más bien por el derecho a rechazar el tratamiento, incluso de soporte vital (eutanasia pasiva); el derecho a los cuidados paliativos, incluso la sedación terminal (eutanasia activa indirecta); el derecho a otorgar el documento 
de instrucciones previas y ciertos derechos prestacionales ligados a la protección de la intimidad (derecho a una habitación individual para el enfermo terminal), a una asistencia específica para el enfermo y su familia, etcétera. Con estos derechos se pretende reforzar la libertad o autonomía del enfermo terminal frente a las decisiones del final de la vida (es una manifestación peculiar del derecho general al consentimiento informado ${ }^{20}$ ), pero también su intimidad en el proceso de morir, así como la protección frente al dolor y la mejora de las condiciones fácticas presentes en dicho proceso.

En mi opinión, si no todo el nuevo conjunto de derechos personales en el proceso final de la vida o en el proceso de morir, sí, al menos, los más importantes aspectos, tienen ya en España estatura constitucional porque se pueden deducir del catálogo de derechos fundamentales de nuestra Constitución. Concretamente, el derecho a rechazar tratamientos de soporte vital y el derecho frente al dolor físico pueden deducirse, sin gran dificultad, del derecho fundamental de integridad personal del artículo 15 de la Constitución. La interpretación de nuestro ordenamiento, ex artículo 10.2 CE, a partir de la Sentencia V.C. contra Eslovaquia, de 8 de noviembre de 2011, permitiría alcanzar tal conclusión. Pero es que ya una Sentencia del Tribunal Constitucional, la STC 37/2011, de 28 de marzo, ha venido a considerar expresamente el consentimiento informado como un derecho fundamental ${ }^{21}$. Según el Tribunal, el consentimiento informado es algo inherente al derecho fundamental de integridad física: «se trata de una facultad de autodeterminación que legitima al paciente, en uso de su autonomía de la voluntad, para decidir libremente sobre las medidas terapéuticas y tratamientos que puedan afectar a su integridad, escogiendo entre las distintas posibilidades, consintiendo su práctica o rechazándola» (incluso aunque pudiera conducir a un resultado fatal). Y en el fundamento jurídico tercero, sostiene que el derecho a la integridad personal comprende «una facultad de oposición a la asistencia médica

20 Derecho que, por cierto, el Tribunal Europeo de Derechos Humanos ha reconocido recientemente como una dimensión del derecho a la integridad personal del artículo 3 del Convenio de Roma en la Sentencia V.C. contra Eslovaquia, de 8 de noviembre de 2011. La demandante era una mujer gitana a la que en un hospital público, tras el parto del segundo hijo por cesárea, y ante los riesgos derivados de un eventual tercer embarazo, se la esteriliza, pero sin solicitarle previamente su consentimiento. El Tribunal considera que esta intervención paternalista habría lesionado su derecho al consentimiento informado, es decir, habría violado su derecho a la integridad personal (artículo 3), pero también aprecia un cierto sesgo discriminatorio por motivos racistas. En efecto, la probabilidad de sufrir este tipo de intervenciones es mayor en relación con las mujeres gitanas, dados los prejuicios racistas existentes en el país y, en particular, la idea de que las mujeres gitanas tienen demasiados hijos.

21 El recurrente alegaba una lesión de su derecho fundamental a la integridad física (artículo $3 \mathrm{CE}$ ) por habérsele denegado el derecho a ser indemnizado por la pérdida funcional de la mano derecha como consecuencia de la realización de un cateterismo cardiaco sin que se le hubiera informado de los riesgos de la intervención ni se hubiera recabado consentimiento para la práctica de la misma. El Tribunal considera que no son consistentes los argumentos mantenidos por los órganos judiciales ordinarios para desestimar la indemnización: la intervención era urgente, el paciente tenía bastante edad y años atrás ya había sufrido una intervención similar. En efecto, el Tribunal observa que el paciente ingresó en urgencias a las 14:16 del 4 de septiembre de 2005 y el cateterismo no se le practicó hasta el día siguiente; y los jueces no demuestran ni la «gravedad» ni la «inmediatez». 
en ejercicio de un derecho de autodeterminación que tiene por objeto el propio sustrato corporal como distinto del derecho a la salud o a la vida».

Por otro lado, el derecho a otorgar un documento de instrucciones previas o voluntades anticipadas también sería deducible de este derecho al consentimiento informado. Es una concreción del mismo cuando el paciente es terminal o sufre una enfermedad grave irreversible y no puede decidir por sí mismo qué medidas adoptar.

Más discutible es que los derechos prestacionales (derecho a una habitación individual o a una asistencia específica para el paciente o los familiares), que en la práctica son muy importantes, formen parte del contenido constitucional esencial. Más bien, parecen ser derechos de estricta configuración legislativa que dependen de las condiciones fácticas y económicas existentes.

En definitiva, el ordenamiento español ofrece en este punto una experiencia hasta cierto punto innovadora que pudiera resultar interesante desde la perspectiva del derecho comparado.

¿QUÉ SIGNIFICA EN EL

ORDENAMIENTO

ESPAÑOL EL

DERECHO A

«VIVIR CON

DIGNIDAD EL

PROCESO DE LA

MUERTE»?

WHAT DOES «THE

RIGHTTO LIVE

THE PROCESS

OF DYING WITH

DIGNITY» MEAN

IN SPANISH LAW? 